\title{
The Periotest Method: Implant-Supported Framework Precision of Fit Evaluation
}

\author{
Kenneth B. May, DDS, * Marion J. Edge, DMD, MEd, $\dagger$ \\ Brien R. Lang, DDS, t and Rui-Feng Wang, BS\$
}

\begin{abstract}
Purpose: In this study, the Periotest instrument was used to measure the precision of fit between cast high noble-metal frameworks and the supporting implants in a patient-simulation model. Three framework conditions and three implant-location variables were used to evaluate the rigidity of the assembly as measured by the Periotest method. The framework variables were (1) one-piece castings (OPC); (2) sectioned-soldered inaccurate castings (SSIC); and (3) sectioned-soldered accurate castings (SSAC). The implant-location variables were right anterior (RA), center (C), and left anterior (LA).

Materials and Methods: The patient simulation model used consisted of three self-tapping Brånemark implants in a reasonable arch curvature in bovine bone. Three working casts were fabricated from the patient-simulation model using polyvinyl siloxane and tapered impression copings. From the working casts, three sets of three frameworks were fabricated as OPCs, SSICs, and SSACs using type 3 high noble alloy. The SSICs were fabricated with a quantitative misfit of $101.6 \mu \mathrm{m}$ at the facial surface, between the abutment-to-gold cylinder interface at the $\mathrm{C}$ implant location. Periotest value (PTV) measurements were made at the midfacial surface of the frameworks directly above each abutment-to-gold cylinder interface. Three measurements were made for each test condition. The data were analyzed to compare framework condition(s) and implant location(s) using ANOVA and Fisher's Protected Least Significant Difference Comparison Test.

Results: The ANOVA showed that significant differences exist between the mean PTV data for framework condition and for implant location $(\rho<.01)$. Significant differences were shown between the mean PTV data for the SSAC assemblies and the OPC and SSIC assemblies. The SSICs displayed a more positive (+) mean PTV than the OPCs. The OPC assemblies had a more positive mean PTV than the SSAC assemblies. The mean PTV data for the SSAC assemblies had a significantly different PTV $(\rho<.01)$ than the other two framework condition assemblies. The OPC and the SSIC assemblies had PTVs that were not significantly different. The $\mathrm{C}$ implant location was significantly different from the RA and the LA implant locations $(p<.01)$. The RA and the LA implant locations were not significantly different from each other. The $\mathrm{C}$ implant location always demonstrated the most positive mean PTV regardless of the framework condition being tested.

Conclusions: The Periotest instrument quantified differences in the precision of fit between three framework conditions. The SSAC assemblies were significantly more rigid than the OPC and SSIC assemblies. The OPC and SSIC assemblies' mean PTVs were not significantly different. The mean PTVs for the C implant location and the RA and LA implant locations were significantly different $(p<.01)$. The mean PTVs of the RA and LA implant locations were not significantly different. The implant-location PTVs followed the same rank order for all three framework conditions. The procedures used to fabricate a more precise fit between the framework and the supporting implants is influenced by the skill of the clinician and technician.
\end{abstract}

JProsthod 1996;5:206-213. Copyright 1996 by the American College of Prosthodontists.

INDEX WORDS: precision, implant, fit, framework, substructure, interface

From the Department of Prosthodontics, School of Dentislny, University of Michigan, Ann Arbor, MI.

*Assistant Professor.

tClinical Assistant Professor.

$\ddagger$ Professor and Chair.

\$Research Associate I.

Accepted March 6, 1996.

Supported in parl by BioResearch, Milceaukee, WT, and the Depariment of Prosthodonitis, University of Michigan School of Dentisty.

This aricle was derived from an oral presentation at the 24 th Annual Scientific Program of The American College of Prosthodontists, October 1993, Palm Springs, CA.

Correspondence to: Kenneth B. May, DDS, Department of Prosthodontics, School of Denissty, University of Michigan, Ann Arbor, MI 48109-1078.

Coppright $\odot 1996$ by the American College of Prosthodontists

$1059-941 X / 95 / 0503-0009 \$ 5.00 / 0$
A $\mathrm{N}$ ACCEPTABLE FRAMEWORK for a fixed Aimplant prosthesis is one that shows a precision of fit between the implant components and the framework casting. ${ }^{1-12}$ However, the lost-wax casting technique may not routinely produce the desired fit, especially in a long-span framework, because precision of fit may be affected by the dimensions of the casting. ${ }^{4-10,13-21}$ In contrast, some clinicians cast the framework in two or three segments and solder or braze the sections together to obtain a framework with the desired precision. ${ }^{4-7}$ In these situations, the clinician generally relies on visual acuity to assess the fit at the abutment-to-framework or implant interface(s). Radiographs have also provided another 
method of evaluating the precision of fit at this interface (Adrian EA, personal communication, July 1992) ${ }^{22-24}$ At best, both of these methods of assessing precision are subjective. Clinicians would prefer an "objective" method that provides quantitative data about the fit or misfit. One such method suggested by Schulte ${ }^{25,26}$ uses the Periotest instrument. The application of the Periotest instrument in implant dentistry has been documented. ${ }^{25-34}$ The ranges of Periotest values (PTVs) have been established for the determination of osseointegration of implants. ${ }^{26,28-32}$ May et al investigated the ability of the instrument to discriminate between accurately assembled implant components in vitro (May et al, unpublished data, September 1992). The investigation showed that the Periotest instrument could discriminate between accurately assembled abutments and gold cylinder using the Brånemark System (Brånemark, Nobelpharma AB, Göteborg, Sweden). In follow-up investigations, May et al used the Periotest instrument to quantify a series of misfits created in abutments and gold cylinders assembled on implants in an in vitro bone model. ${ }^{33}$ Significant differences were found within and between component groups. The Periotest instrument was evaluated for its ability to discriminate differences in framework assemblies as influenced by retaining screws tightening sequences for groups of accurate and inaccurate framework assemblies. The investigation found that there were no significant differences in fit of the framework assemblies with each framework condition. No differences were found regardless of the tightening sequence used within each framework condition, but there were significant differences between the accurate and inaccurate framework groups. ${ }^{34}$ These findings provide additional support of the validity, specificity, and repeatability of the instrument.

Hence, the Periotest instrument may also provide an accurate method to assess the fit of an implantsupported framework. One would expect the PTVs to be more negative (-) under conditions of a rigid assembly that results when a precision of fit exists between the abutment and the framework. However, in the presence of a less rigid assembly that exists when a precision of fit is lacking, the Periotest instrument would record more positive $(+)$ values. This study was initiated to test this theory and to determine if the Periotest instrument was sensitive enough to measure differences between frameworks that show a precision fit and various misfit conditions. Specifically, the study would quantify the fit between the implant abutments for a patient simulation consisting of three implants, and (1) the onepiece casting (OPG), (2) a sectioned-soldered inaccurate casting (SSIC), and (3) a sectioned-soldered accurate casting (SSAC). The null hypotheses are (1) no statistically significant differences exist between the three framework conditions at the abutment-toframework interfaces as measured by the Periotest method, and (2) no statistically significant differences exist between the three implant locations for a framework condition as measured by the Periotest method.

\section{Materials and Methods}

The patient simulation model consisted of three selftapping implants (Brănemark) (SDCA 062; $3.75 \times 10.0$ $\mathrm{mm}$ ) positioned cqual distances $(7-10 \mathrm{~mm})$ apart in bovine bone in a reasonable curvature (right anterior $[\mathrm{RA}]$, center [C], and left anterior [LA]) for an implant-supported prosthesis. Abutments $5.5 \mathrm{~mm}$ in length were assembled on the implants with the center screws torqued to $20 \mathrm{~N}-\mathrm{cm}$ using the Brånemark hand-torque controller. Radiographs of the implant-to-abutment interfaces were made to ensure proper assembly. The absence of any radiographic gap at the interface was interpreted as a precision of fit. The PTVs werc recorded at the midfacial surface of the three abutments following assembly.

Tapered impression copings (DCA 106) were attached to the implant abutments on the patient simulation model, and impressions were made using a custom impression tray (Fast-tray; Bosworth Co, Skokie, IL) and polyvinyl siloxane (Extrude; Kerr, Romulus, MI) to produce a master cast. After making the impression, brass replicas (DCA 015) were joined to the impression copings, the copings were repositioned in the final impression, and the impression was poured in gypsum stone (Jade Stone; Whip-Mix Corp, Iouisville, KY). Three separate impressions were made using this technique to produce three master casts. The master casts were allowed to stand for a minimum of 36 hours before initiation of the procedures to construct the cast frameworks.

The OPC frameworks (Fig 1) were made using the acrylic wing components (DCA 100) fitted to the center brass replicas on each of the three master casts. The wings on either side of the center abutment were cut to the proper length and waxed to individual Brånemark gold cylinders (DCA 072) positioned on the right and left brass replicas. The OPC patterns were removed from the master casts and invested in a phosphate-bonded investment (Whip-Mix Corp). The burnout procedure was conducted according to the manufacturer's instructions. The frameworks were cast in a type 3 high noble alloy (Option, Ney Co, Bloomfield, CT). The cast frameworks were recovered from the investment, cleansed of debris using an alumina 


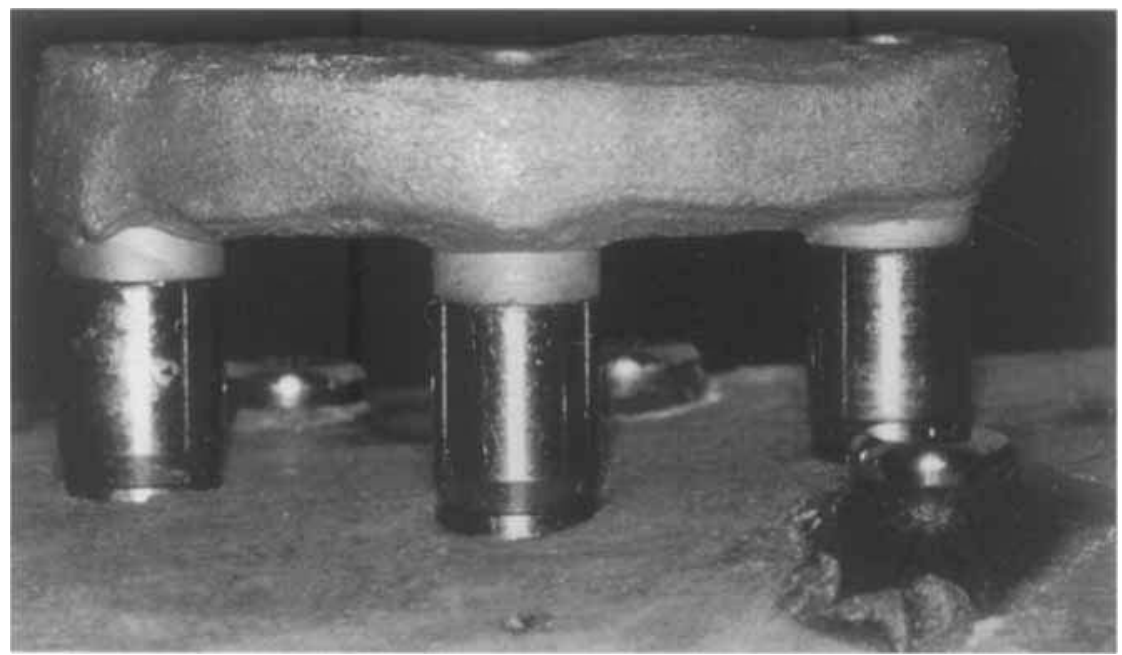

Figure 1. The OPG framework passively seated on the patient simulation model.

air abrasive, and pickled in an acid solution (Prevox; Whip-Mix Corp). The three OPC frameworks were positioned on the patient simulation model using long guide pins by tightening the center, right, and left guide pins in that order. The guide pins were torqued to $10 \mathrm{~N}-\mathrm{cm}$. Periotest measurements were made at the midfacial surface of the framework directly above the abutment-to-gold cylinder interface (Fig 2). Three assembly and measurement replications were made for each of the OPC frameworks for a total of nine measurements per framework.

The OPC frameworks were removed from the patient simulation model and sectioned into three segments by cutting midway between the gold cylinders, in preparation for reassembly and soldering in an inaccurate relation (SSIC). The right and left segments were accurately positioned on the patient simulation model. The center segments, however, were positioned with a 101.6- $\mu \mathrm{m}$ (0.004-in) thickness gauge (L.S. Starrett Co, Athol, MA) inserted between the abutment and the gold cylinder in

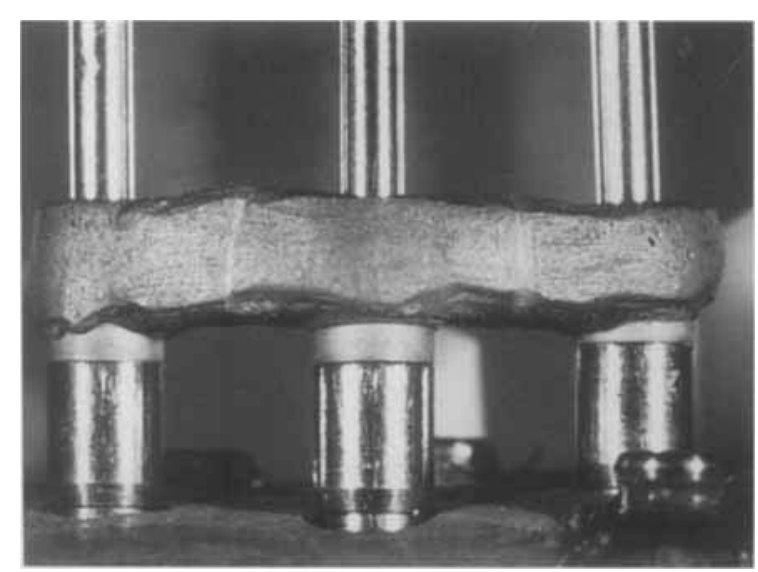

Figure 2. The assembled OPC framework on the patient simulation modcl being measured with the Periotest instrument. the facial area to create a quantitative misfit at the interface. The guide pins were torqued to $10 \mathrm{~N}-\mathrm{cm}$. The segments were luted together before soldering using a chemically polymerized acrylic resin (Duralay; Reliance Dental Mfg Co, Worth, IL), removed from the patient simulation model, invested in a high-heat solder investment (Biovest; Dentsply Int, Inc, York, PA), and soldered in a conventional manner using gold solder (Ney 650; Ney $(\mathrm{Co})$.

The SSIC frameworks (Fig 3) were recovered, cleaned, and placed on the patient simulation model using the guide pins torqued to $10 \mathrm{~N}-\mathrm{cm}$, following the same tightening sequence outlined previously. Periotest measurements were conducted at each implant site for each of the frameworks. Threc measurement replications were made for each of the three frameworks for a total of nine measurements per framework.

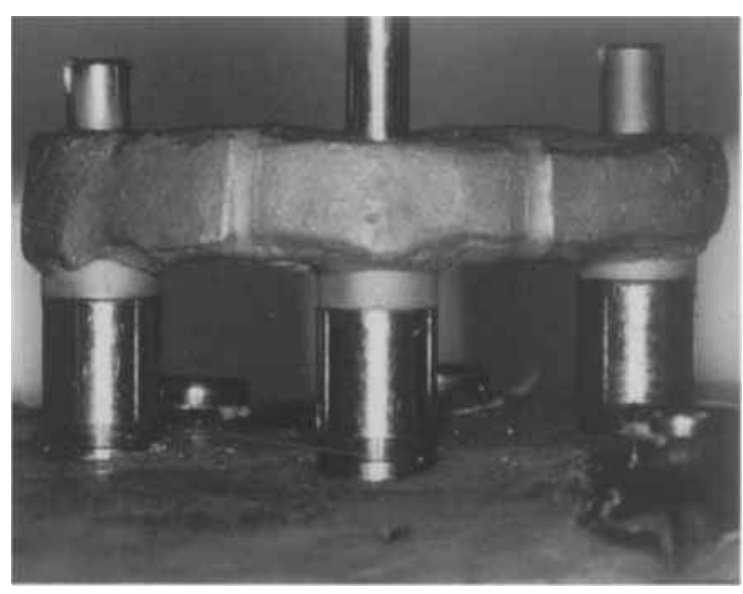

Figure 3. The SSIC framework assembled on the patient simulation model demonstrating the presence of a gap at the abutment-to-gold cylinder interface on the buccal of the center implant location. 
The SSIC frameworks were removed from the patient simulation model and re-sectioned through the present solder joints between the gold cylinders, in preparation for reassembly and soldering in an accurate condition (SSAC). All thrce segments were accurately positioned on the patient simulation model, and the guide pins were torqued to $10 \mathrm{~N}-\mathrm{cm}$. The segments were luted together, removed from the patient simulation model, and soldered in the accurately assembled condition.

The SSAC frameworks (Fig 4) were recovered, cleansed, and positioned on the patient simulation model for Periotest measurements. Three measurement replications were repeated for the three accurate frameworks, for a total of nine measurements per framework.

The analysis of the collected PIV measurements consisted of (I) descriptive statistics, (2) a two-way ANOVA ${ }^{34,35}$ for the changes in PTVs between the three framework conditions at the three implant locations, and between the three implant locations within a framework condition, and (3) Fisher's Protected Least Significant Difference ${ }^{37}$ (LSD) pairwise comparisons.

\section{Results}

A two-way ANOVA was conducted on the PTVs recorded to determine if significant differences existed between the framework conditions and the implant locations when (1) all three implant locations were considered (RA, C, and LA), and (2) the C location was removed from the data set and only $\mathrm{RA}$ and IA locations were considered (Table 1). The data showed that there were no significant differences between the three frameworks evaluated $(p=.1835$ and $p=.2565)$ and the three measurements of each framework $(p=.6906$ and $p=.7767)$.

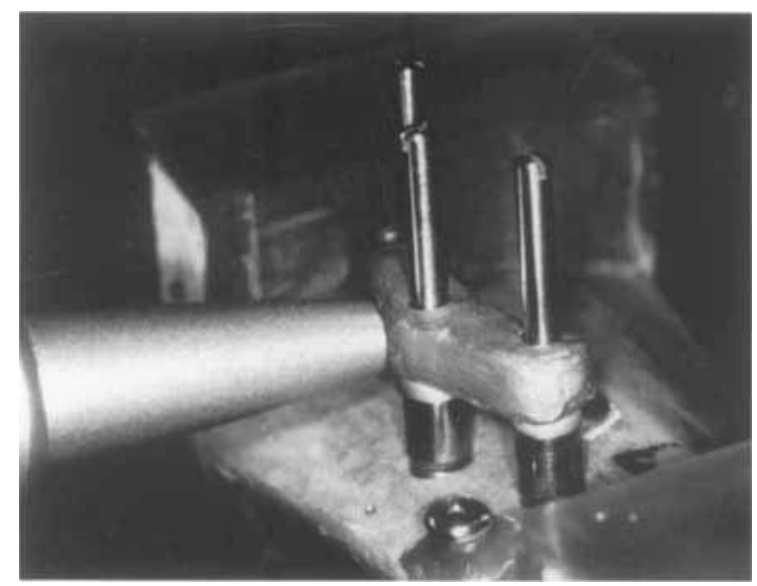

Figure 4. The SSAC framework assembled on the patient simulation model demonstrating the absence of a gap between the abutment-to-gold cylinder interface of the supporting implants.
Table 1. The Two-Way ANOVA of the Framework Conditions and the Implant Locations Compared by Three and by Two Implant Locations

\begin{tabular}{|c|c|c|c|c|}
\hline \multirow[b]{2}{*}{ Source } & \multicolumn{2}{|c|}{$\begin{array}{l}\text { Locations } \\
\text { (3) }\end{array}$} & \multicolumn{2}{|c|}{$\begin{array}{l}\text { Locations } \\
\text { (2) }\end{array}$} \\
\hline & $d f$ & $p$ & $d f$ & $p$ \\
\hline Framework condition & 2 & $.0001 *$ & 2 & $.0001 *$ \\
\hline Implant location & 2 & $.0001^{*}$ & 1 & .8168 \\
\hline Framework* implant location & 4 & .2047 & 2 & .9472 \\
\hline Replicate frameworks & 2 & .1835 & 2 & .2565 \\
\hline Replicate measures & 1 & .6906 & 1 & .7767 \\
\hline Replicate frameworks* & & & & \\
\hline Replicate measures & 2 & .8365 & 2 & .5695 \\
\hline Residual & 67 & & 43 & \\
\hline
\end{tabular}

* Significantly different at $p<.01$.

Therefore, the database was judged as reliable. The data also showed $p$ values $(p<.05)$ for the framework conditions when both the two and three implant locations were considered. However, no significant differences were found when the $\mathrm{G}$ location data were removed from the analysis; nor were significant differences found when any of the other combinations of variables were compared.

The means and SDs of the PTV data recorded for the three framework conditions at the three implant locations (RA, C, and LA) are reported in Table 2. The OPC and SSIC frameworks' mean PTVs were significantly different from the mean PTVs recorded for the SSAC framework at the RA implant location $(p<.05)$. Similar differences were found at the $\mathrm{C}$ location and the LA location for the OPC and SSIC frameworks.

The means and SD of the PTV data recorded at the three implant locations (RA, C, and LA) are reported for the three framework conditions tested

Table 2. The Means and SD of the Framework Conditions at the Three Implant Locations

\begin{tabular}{clc}
\hline \multicolumn{1}{c}{ Condition } & Mean $(S D)$ & $p$ \\
\hline RA implant location & & \\
OPC & $-6.6(1.2)^{*}$ & $.0001^{*}$ \\
SSAC & $-8.0(0.0)$ & \\
SSIC & $-6.4(0.5)^{*}$ & \\
G implant location & & \\
OPC & $-3.4(3.4)^{*}$ & $.0005^{*}$ \\
SSAC & $-6.4(2.5)$ & \\
SSIC & $-2.4(4.2)^{*}$ & \\
LA implant location & $-6.6(0.9)^{*}$ & $.0001^{*}$ \\
OPC & $-8.0(0.0)$ & \\
SSAC & $-6.3(0.7)^{*}$ & \\
SSIC &
\end{tabular}

* Significantly different at $P<.05$. 
in Table 3. In the OPC, there was a significant difference in the PTVs at the RA and LA locations in comparison with the $\mathrm{C}$ location. This observation was also found in the SSIC and SSAC frameworks $(p<.05)$.

The mean PTVs for the three locations were compared within each framework conditions (Table 4). No significant differences were found when RA was compared with LA in the OPC, SSIC, or SSAC frameworks. However, significant differences were found when RA and LA locations were compared with the $\mathrm{C}$ location in all three framework conditions $(p<.05)$.

The mean PTVs for the three framework conditions were compared with each other at the three implant locations (Table 5). Significant differences were found when the SSAC frameworks were compared with the OPC and SSIC frameworks at all three implant locations $(p<.05)$. No significant differences were found when the OPC and SSIC data were compared.

\section{Discussion}

A critical step in implant-supported prostheses used in rehabilitations is the verification of the precision of fit of the framework. Traditionally, frameworks used in implant-supported rehabilitations are fabricated as either one-piece castings ${ }^{13-17,21}$ or sectionedsoldered castings. ${ }^{4-7,18-20}$ The method used to correct the fit, should a misfit occur, is to section the casting, lute the sectioned segments together with an autopolymerizing resin intraorally, and solder the framework sections. The framework is then returned to the master cast and the offending abutment replica(s) is corrected. The acceptability of a framework is judged

Table 3. The Means and SD of the Implant Locations Compared by the Framework Conditions

\begin{tabular}{clc}
\hline Location & Mean $(S D)$ & $p$ \\
\hline OPC $(\mathrm{n}=3)$ & & \\
RA & $-6.6(1.2)^{*}$ & $.0004^{*}$ \\
C & $-3.4(3.4)$ & \\
LA & $-6.6(0.9)^{*}$ & \\
SSIC $(\mathrm{n}=3)$ & & \\
RA & $-6.4(0.5)^{*}$ & $.0006^{*}$ \\
C & $-2.4(4.2)$ & \\
LA & $-6.3(0.7)$ & \\
SSAC $(\mathrm{n}=3)$ & & \\
RA & $-8.0(0.0)^{*}$ & $.0328^{*}$ \\
C & $-6.4(0.8)$ & \\
LA & $-8.0(0.0)^{*}$ & \\
\hline
\end{tabular}

*Significantly different at $p<.05$.
Table 4. Fisher's Protected LSD Comparison Test of the Implant Location by Framework Condition

\begin{tabular}{clll}
\hline & \multicolumn{3}{c}{$p$} \\
\cline { 2 - 4 } Location & \multicolumn{1}{c}{$O P C$} & $S S I C$ & $S S A C$ \\
\hline RA vs LA & 1.0000 & .9094 & 1.0000 \\
vs C & $0.0004^{*}$ & $.0005^{*}$ & $0.0225^{*}$ \\
LA vs C & $0.0004^{*}$ & $.0007^{*}$ & $0.0225^{*}$ \\
\hline
\end{tabular}

*Significantly different at $p<.05$.

clinically by visual and tactile evaluations for the presence or absence of a gap between the framework and the implant. The evaluation may also include a radiographic survey to aid in determining the presence of a gap (Adrian EA, personal communication, 1992). ${ }^{22-24}$ However, at best, these methods possess subjective clinical and technical qualities.

Schulte ${ }^{26}$ has suggested that the Periotest instrument can be used to detect differences in the adaptation of the retainers for a fixed partial denture to the prepared abutment teeth, and thus provide a quantitative value for the fit. Similarly, this instrument was evaluated in the present in vitro investigation as a method to assess the precision of fit between the framework and the supporting implants. This study addressed the questions dealing with the influence of the framework conditions, ie, the one-piece condition, the solder-inaccurate condition, and the soldered-accurate condition, on the quantitative PTVs recorded at the various implant locations supporting these frameworks.

In Table 2, the OPC and SSIC framework assemblies demonstrated significant differences in the PTVs at all locations, while the SSAC framework assembly did not. The mean PTVs for both the OPC and the SSIC assemblies were less negative than those for the SSAC. The less negative PTVs were interpreted as meaning that a better fit existed between the mating supporting interfaces of the frameworks and the implants with the SSAC framework than the other two framework conditions. Both the OPC and SSIC frameworks lacked the accuracy in fit that was observed with the SSAC framework as measured by the Periotest instrument, thus rejecting

Table 5. Fisher's Protected LSD Comparison Test for Differences in the Framework Conditions

\begin{aligned} & \hline Conditions \multicolumn{1}{c}{$p$} \\ & \hline SSAC vs OPC $.0001^{*} \\ &$ vs SSIC $.0001^{*} \\ &$ OPC vs SSIC .3408 \\ & \hline\end{aligned}

*Significantly different at $p<.01$. 
the first null hypothesis of the expcriment that "no statistically significant differences exist between the three framework conditions at the abutment-toframework interfaces as measured by the Periotest Method." However, the literature indicated equivalence and differences in the framework fit depending on whether the OPC or the SSAC method of framework fabrication is utilized. ${ }^{4-10,13-21}$ The SSAC framework is the most accurate of the three framework designs used in this study and supports the sectioned method of framework fabrication. The Periotest instrument has been shown to reproducibly discriminate various degrees of firmness or mobility at the implant-bone interface. ${ }^{26-31}$ Also, differences within and between accurately (May et al, unpublished data, September 1992) and inaccurately ${ }^{33,34}$ assembled implant components were shown through in vitro investigations using the Periotest instrument.

The mean PTV for the $\mathrm{C}$ position was more negative for the SSAC framework condition than the other two framework conditions tested. However, the mean PTVs did not reach the -8.0 value that was observed in the RA and LA locations for the SSAC frameworks. This effect was probably the result of minor inaccuracies in the soldering procedure. Thus, the technical skill of the laboratory personnel may be an issue, as observed in conventional prosthodontic rehabilitations.

In Table 3, the PTVs for location C demonstrated significant differences with respect to the PTVs for the other implant locations (RA and LA) for all three framework conditions $(p<.05)$. In comparing the OPC and the SSIC frameworks' mean PTVs, the OPC framework's data demonstrated mean PTV data analogous with better stability or a more rigid assembly. The greatest magnitude of change in mean PTV data occurred at the $\mathrm{C}$ implant location, where the SSIC mean PTV was more positive by a value of 1.0 PTV when compared with the OPC. The SSAC framework's mean PTV data were the most negative for all implant locations when compared with the other two framework conditions (-8.0, -6.4 , and -8.0 ). It would appear that the SSAC framework condition demonstrated the most accurate fit of the framework conditions tested.

Overall, the $\mathrm{C}$ implant location continued to demonstrate a slightly more positive mean PTV in comparison with the two other implant locations. The ANOVA (Table 3) demonstrates that significant differences exist in the implant locations $(p<.05)$. Even the accurate fabrication technique resulted in some inaccuracy, because the $\mathrm{C}$ implant location was inherently more positive in mean PTV. The observance of significant differences at the various implant locations under the different framework conditions leads to the rejection of the second null hypothesis that "no statistically significant differences exist between the three implant locations for a framework condition as measured by the Periotest Method." The Periotest instrument is able to quantitatively detect differences in precision of fit.

Misfits between a framework and the supporting implants have many etiologies. Factors such as type of alloy, framework fabrication technique, implant (abutment) lengths, implant angulations, and the distance between the implants are among the most common. Assembly inconsistencies in the torquing sequences during framework attachment to the implants is another factor to be considered. However, of the previously listed factors, the framework fabrication process is probably the most common cause and has the greatest potential for causing errors or misfits of a larger magnitude. From this study, the OPC frameworks appeared to have a better precision of fit than the SSIC frameworks, yet far worse than the SSAC frameworks (Tables 2, 3).

The SSIC frameworks were fabricated with a quantitative misfit of $101.6 \mu \mathrm{m}$ on the buccal of the $\mathrm{C}$ implant location at the abutment-to-framework interface. A misfit in each framework translated into a more positive shift in the mean PTV data when compared with the OPC and the SSAC frameworks. These findings support the use of the Periotest instrument in the clinical setting as a device to detect misfits of approximately $0.1 \mathrm{~mm}$. Whether the instrument is able to detect misfits smaller than the $0.1-\mathrm{mm}$ gap and demonstrate greater sensitivity needs further study. An cqually important finding in this project was the discovery that the OPC framework assemblies had more negative PTVs than the SSIC framework assemblies, but not as negative as the SSAC framework assemblies. Thus, this means that the fabrication of frameworks in sections and soldering produces a better precision of fit than casting the framework as one piece, as reported in the literature. However, the SSAC frameworks were not without flaw. The data from the three framework fabrication conditions, including a quantified misfit, supported previous investigations, ${ }^{13-21}$ which indicate the scnsitivity of framework fabrication. Hence, the fabrication procedure must be conducted with a high level of skill to ensure a precision of fit.

Periotest values of -8.0 should indicate to the clinician that excellent rigidity or unification exists 
between the bearing surfaces of the implants and those of the framework during the assembly procedure.

\section{Conclusions}

Within the limitations of this in vitro study using nonosseointegrated implants placed in a bovine bone model, the framework assemblies were compared for rigidity in the assemblies using the Periotest method. The following conclusions were made:

1. The Periotest instrument is capable of detecting differences in the precision of fit at the implant-toframework interface of three cast high noble alloy framework conditions.

2. The SSAC assemblies measured the most negative PTVs, which is analogous to a more precise fit or more rigid assembly.

3. The SSIC assemblies measured the most positive PTVs, which is analogous to a compromised fit or less rigid assembly, as measured by the Periotest method.

4. An inaccurate fit of a framework at a single bearing surface interface will compromise the rigidity of the framework's assembly, as measured with the Periotest method.

5. The implants demonstrated different mean PTVs depending on the implant location, as measured by the Periotest method.

6. The procedures used to fabricate a more precise fit between the framework and the supporting implants is influenced by the skill of the clinician and technician.

\section{References}

1. Rangert B, Jemt T, Jorneus L: Forces and moment on Brånemark implants. Int J Oral Maxillofac Implants 1989;4: 241-247

2. Andersson M: Unik metof for framstallning av kronersattningar i titan. Tandlakartidingen 1987;79:640-642

3. Adell R, Eriksson B, Lekholm U, et al: A long-term follow-up study of osseointegrated implants in the treatment of totally edentulous jaws. Int J Oral Maxillofac Implants 1990;5:347359

4. Brånemark P-I, Zarb GA, Albertsson T: Tíssue-integrated prostheses. Osseointegration in clinical dentistry. Chicago, IL, Quintessence Publishing Co, 1985, p 303

5. McCartney J: Intraoral connection of individual abutment attachments for an osseointegrated implant-supported prosthesis. J Prosthet Dent 1991;66:799-803

6. Balshi TJ, Fox N: Cast framework design for fixed prosthodontics supported by Bjotes osseointegrated implants. Trends and Techniques 1986;3:32-35
7. Voitik AJ: The new type full mouth rehabilitation, Trends and Techniques 1989;9:30-37

8. Voitik AJ: The Kulzer abutment luting; KAL Technique a dircct assembly framework method for osseointegrated implant prostheses. The Implant Society 1991;2:11-14

9. Sellers GC: Direct assembly framework for osseointegrated implant prosthesis.J Prosthet Dent 1989;62:662-668

10. Jemt $\mathbf{T}$ : Modificd single and short-span restorations supported by osseointegrated fixtures in the partially edentulous jaw.J Prosthet Dent 1986;55:243-246

11. Worthington P, Bolender CL, Taylor TD: The Swedish system of osseointegrated implants: problems and complications encountered around a 4-ycar trial period. Int $J$ Oral Maxillofac Implants 1987;2:77-84

12. Richter EJ: Basic biomechanics of dental implants in prosthetic dentistry. J Prosthet Dent 1989;61:602-609

13. Bruce RW: Evaluation of multiple unit castings for fixed partial dentures. J Prosthet Dent 1964;14:939-943

14. Fusayama T, Wakumoto $S$, Hosada H: Accuracy of fixed partial dentures made by various soldering techniques and one piece casting.J Prosthet Dent 1964;14:334-342

15. Huling JS, Clark RE: Comparative distortion in three unit fixed prosthesis joined by laser welding, conventional soldering, or casting in one piece.J Prosthet Dent 1977;56:128-134

16. Saas FA, Eames WB: Fit of unit cast fixed partial dentures related to casting ring size and shape. J Prosthet Dent 1980;43:163-167

17. Garlapo D, Lee S-H, Choung CK, et al: Spatial changes occurring in fixed partial dentures made as one-piece castings. J Prosthet Dent 1983;49:781-785

18. Schiffleger BE, Ziebert GJ, Dhuru VB, et al: Comparison of accuracy of multiunit one-piece castings. J Prosthet Dent 1985;54:770-776

19. Ziebert GJ, Hurtado A, Glapa C, et al: Accuracy of one-piece castings, preceramic and post-ceramic soldering. J Prosthet Dent 1986;55:312-317

20. Sarfati E, Harter J-C: Comparative accuracy of fixed partial dentures made as one-piece casting or joined by solder. Int $\mathrm{J}$ Prosthodont 1992;5:377-383

21. Tan KB, Rubenstein JE, Nicholls JI, et al: Three-dimensional analysis of the casting accuracy of one-piece, osseointegrated implant-retained prostheses. Int J Prosthodont 1993;6:346363

22. Binon PP: The Annual Session of the American Academy of Osseointegration. San Diego, CA, 1993

23. Hollender L: Radiographic techniques for prccision analysis of bridges on osseointegrated fixturcs. Swed Dent J 1985(suppl 28): $171-174$

24. Cox JF, Pharoah M: An alternative holder for radiographic evaluation of tissue-integrated prostheses. J Prosthet Dent 1986;56:338-341

25. Schulte W: A new field of application of the Periotest method. The occlusal-periodontal load can now be measured quantitatively. Zahnarztl Mitt 1988;78:1-11

26. Schulte W: The Periotest Method. Tubingen, Special Publication from the Deutscher Zahnarztekalender, 1989:3-14

27. Kohno S, Sato T, Tabata T: Periotest: a new measuring instrument of the dynamic periodontal function and a guide to its application.JPN Quintessence Int 1987;2:41

28. Buser DA, Weber HP: Evaluation of dental implant mobility using the Periotest procedure (abstr). J Dent Res 1990;69:133 
29. Olive J, Aparicio C: The Periotest method as a measure of osseointegrated oral implant stability. Int J Oral Maxillofac Implants 1990;5:390-400

30. Teerlinck J: Periotest: an objective clinical diagnosis of bone apposition toward implants. Int J Oral Maxillofac Implants 1991;6:55-61

31. Ortman L, Chavez H, Medige J: The assessment of implant mobility (abstr). J Dent Res 1992;71:144

32. Manz MC, Morris HF, Ochi S: An evaluation of the Periotest system. Part I. Examiner reliability and repeatability of readings. Implant Dent 1992;1:142-146

33. May KB, Russell MM, Edge MJ, et al: Precision of Fit at the Implant Prosthodontic Interface. Presented at the 75th meet- ing of The Academy of Prosthodontics, Vancouver, BC (in revision)

34. May KB, Lang BR: The Periotest method: tightening sequence of the retaining screws for fixed/remote implant supported prostheses (abstr). J Dent Res 1995;74:553

35. Neter J, Wasserman W, Kutner MH: Two-factor Analysis of Variancc-Equal Sample Sizes, in Applicd Linear Statistical Models (ed 3). Boston, MA, Irwin, 1990, pp 673-729

36. NeterJ, Wasserman W, Kutner MH: Analysis and Planning of Two-factor Studies Equal Sample Sizes, in Applied Linear Statistical Modcls, (ed 3). Boston, MA, Irwin, 1990, pp 730-760

37. Abacus Concepts: SuperANOVA: Fisher's Protected Least Significant Difference, in SuperANOVA ${ }^{\text {tixim. }}$. Berkeley, CA, Abacus Concepts, 1989, pp 208-209 